\title{
Publisher's Note: Complementarity of quantum correlations in cloning and deleting of quantum states [Phys. Rev. A 91, 062311 (2015)]
}

Sk Sazim, Indranil Chakrabarty, Annwesha Dutta, and Arun K. Pati

(Received 26 July 2016; published 29 July 2016)

DOI: 10.1103/PhysRevA.94.019906

This paper was published online on 9 June 2015 with a typographical error in the author list. The third author's name should read as "Annwesha Dutta." The author name has been corrected as of 22 July 2016. The author name is incorrect in the printed version of the journal. 